\title{
A rare case of invasive micropapillary carcinoma of the breast
}

\author{
Georgios-Ioannis Verras ${ }^{1}$, Francesk Mulita ${ }^{1}$, Levan Tchabashvili', Ioanna-Maria Grypari², \\ Sofia Sourouni ${ }^{3}$, Evangelia Panagodimou ${ }^{4}$, Maria-loanna Argentou ${ }^{1}$
}

1Department of Surgery, Breast Unit, General University Hospital of Patras, Greece

2Pathology Department, University Hospital of Patras, Greece

${ }^{3}$ Department of Radiology, University Hospital of Patras, Greece

${ }^{4}$ Department of Gynecology and Obstetrics, University Hospital of Patras, Greece

\begin{abstract}
Invasive micropapillary carcinoma (IMPC) is a rare, distinct histological subtype of breast carcinoma. While micropapillary histological architecture is found in up to $2-8 \%$ of all breast cancers, pure micropapillary carcinoma is infrequent and comprises $0.9-2 \%$ of breast carcinomas. Invasive micropapillary carcinoma is emerging as an oncological and surgical challenge due to a plethora of characteristics that constitute this histological pattern - interestingly, both elusive and aggressive. We present the case of a woman presenting with IMPC, who was primarily treated with tumour and lymph node marking, followed by primary systemic therapy (PST), and consequent oncoplastic surgery with sentinel lymph node biopsy. Our case report outlines the importance of awareness of histological subtypes in breast cancer by focusing on a case report of IMPC. The breast surgeon must be aware of the lymphotropic behaviour of this subtype and the high prevalence of lymph node involvement in such patients, and therefore focus on rigorous axillary assessment. One must not forget that, despite having a more aggressive biological profile, IMPC has demonstrated no difference in survival when compared to other histological subtypes, and treatment should conform to international guidelines with an emphasis on nodal staging.
\end{abstract}

Key words: breast, carcinoma, invasive, micropapillary.

\section{Introduction}

Invasive micropapillary carcinoma (IMPC) is a rare, distinct histological subtype of breast carcinoma. First described as an entity by Fisher et al. in 1980 [1], it was not until 1993 that the term and classification was introduced by Siriaunkgul et al. [2]. While micropapillary histological architecture is found in up to $2-8 \%$ of all breast cancers, pure micropapillary carcinoma is infrequent and comprises $0.9-2 \%$ of breast carcinomas [3]. The mean age of diagnosis is 59 years, and it is predominantly found in females, with only a few case reports for male IMPC in existence [4-6].

In this report, we present the case of a woman presenting with IMPC, who was primarily treated with tumour and lymph node marking, followed by primary systemic therapy (PST), and consequent oncoplastic surgery with sentinel lymph node biopsy (SLNB). We also provide a summary of current literature regarding the pathology, diagnostics, treatment options, and prognosis of IMPC.

\section{Case presentation}

A 52-year-old postmenopausal woman presented at the Breast Clinic on 3 June 2020 complaining of a palpable mass in the upper-outward quadrant of her right breast. The woman was otherwise healthy, reported no pain, without skin retractions, nipple inversion, or nipple discharge. The patient's medical history was otherwise unremarkable, with negative family history for breast malignancy. Physical examination revealed a non-tender mass in the right breast, lacking clear border definition. Palpation of the axillary lymph nodes also showcased no suspicious masses, classifying the patient as clinically node negative.

Screening mammography of the right breast showed only an enhanced shadowing, compared to her left breast. Mammography tomosynthesis (Fig. 1) revealed a mass lesion, with irregular borders, causing architectural distortion, measuring $1.5 \mathrm{~cm}$ at its maximum diameter.

Ultrasound (U/S) evaluation of the mass (Fig. 2) revealed a hypoechoic lesion of the same dimensions, with posterior acoustic shadowing and increased elasticity on shear wave elastography. Concurrent ultrasonographic evaluation of the axilla yielded a solitary, suspicious level I lymph node, with a measured cortical thickening of more than $3 \mathrm{~mm}$ (Fig. 3).

Core biopsy under U/S guidance was scheduled upon study of the imaging findings. The pathology report came back positive for grade II IMPC. Hormonal recep-

Corresponding author:

Francesk Mulita, MD, Department of Surgery, Breast Unit, General University Hospital of Patras, Greece,

Submitted: 23.10 .2021

e-mail: oknarfmulita@hotmail.com

Accepted: 01.12 .2021 

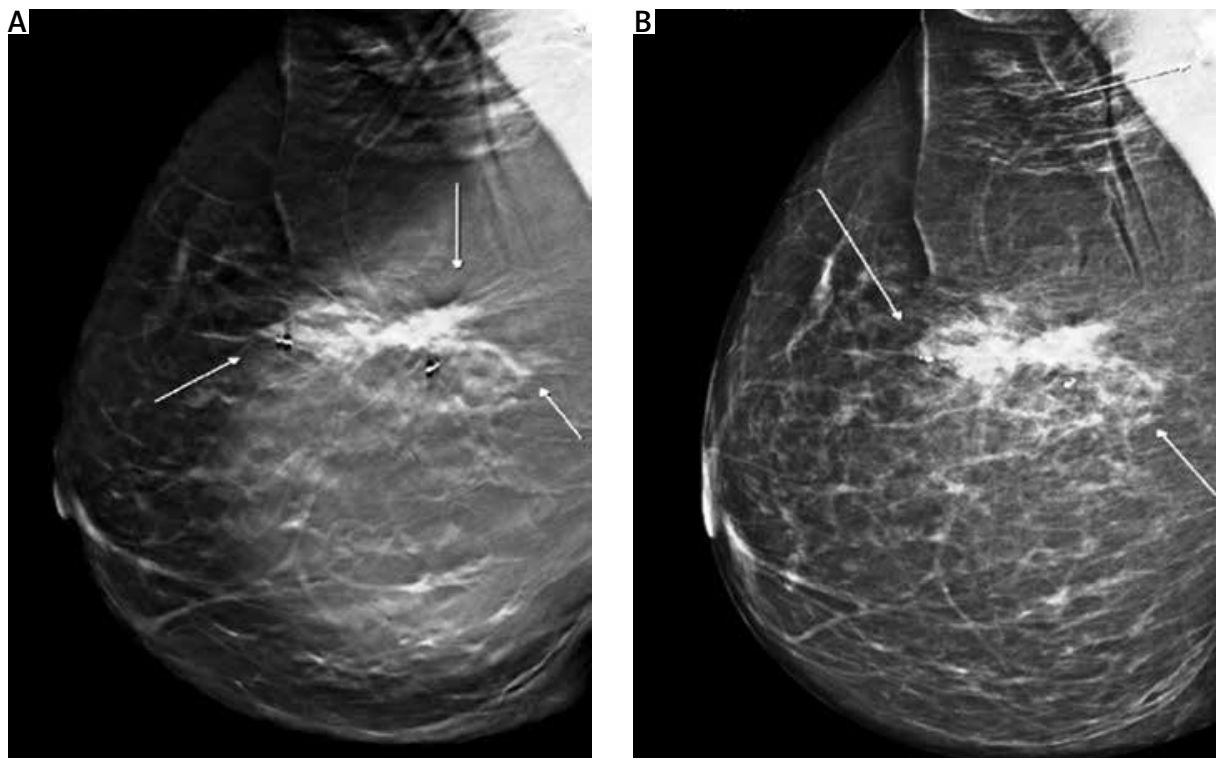

Fig. 1. Mammographic imaging of the lesion. Mass lesion causing architectural distortion at the upper-outer quadrant of the right breast. White arrows indicate the lesion's irregular borders. Biopsy markers are also visible (A, B)
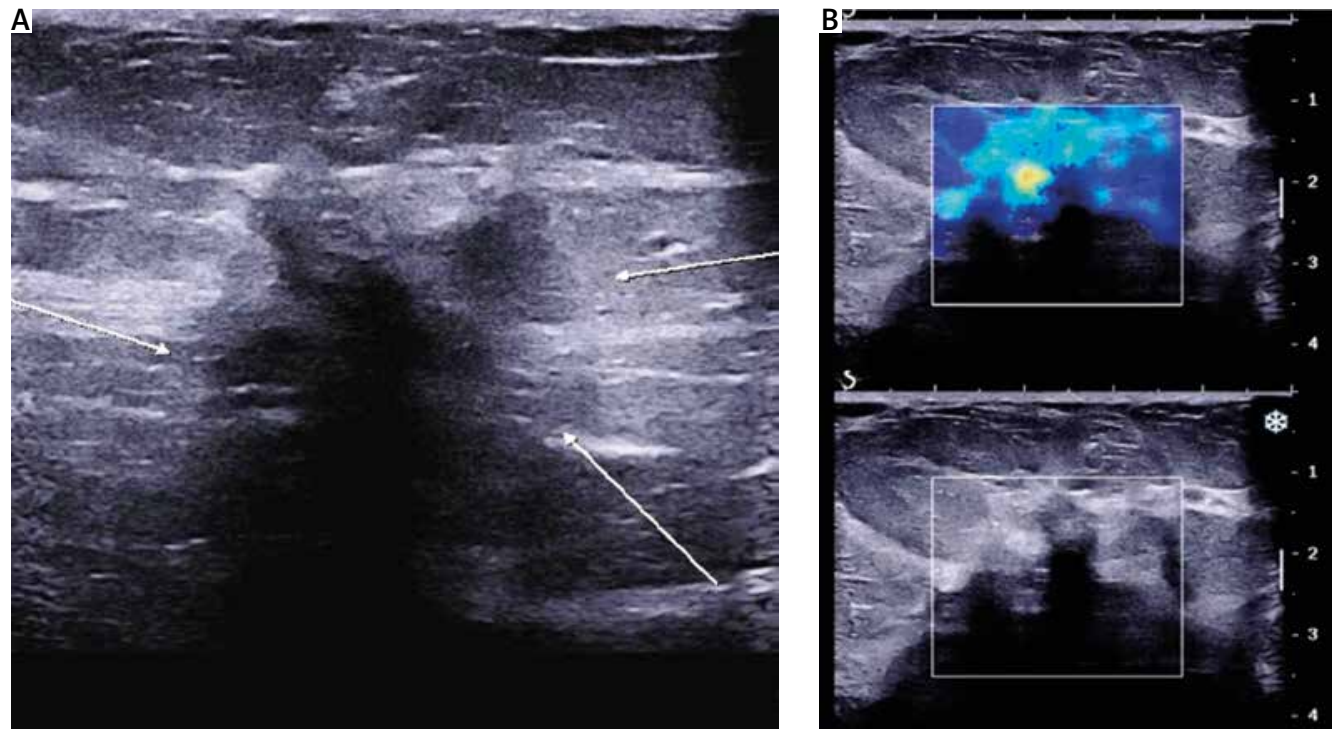

Fig. 2. Ultrasound imaging of the lesion. A) Hypoechoic lesion with indistinct borders (white arrows), posterior acoustic shadowing; B) increased elasticity on shear wave elastography

tor staining and Ki67 index were as follows: oestrogen receptor $-70 \%$, progesterone receptor $-70 \%$, growth factor receptor 2 - score $1+$ (negative), and Ki67 - 30\%. Pathology slides of the tumour are shown in Figures 4-7.

Following the local staging process for patients with breast cancer, the woman was also evaluated with breast magnetic resonance imaging (MRI). Mass and non-mass pathological enhancement, satellite nodules, indistinct shape, and pathological apparent diffusion coefficient values in diffusion weighted imaging, were all in favour of malignancy. The most peculiar finding, however, was the concentric enhancement, around the central lesion, that had a total diameter of $5.2 \mathrm{~cm}$, indicating an extensive lesion that was not visible on $\mathrm{U} / \mathrm{S}$ or mammography (Figs. 7 A-D).
Due to the known predisposition of the micropapillary carcinoma to infiltrate lymphatic routes, the extensive non-mass enhancement was attributed to local lymphatic infiltration. According to the patient's staging, the tumour was $>5 \mathrm{~cm}$, or $\mathrm{T} 3$, and therefore the patient was treated with PST prior to surgery, according to the current oncological guidelines. Considering the patient's advanced disease stage, postmenopausal status, and hormonal positivity, the oncological consultant decided upon administration of preoperative hormonal therapy in the form of an aromatase inhibitor (anastrozole) for a duration of 6 months.

Owing to the unusual imaging of the mass and the decision for the patient to undergo PST prior to surgery, based upon the MRI findings, biopsy markers were 
placed under U/S guidance - one in the centre of the mass and the other in the surrounding non-mass enhanced area (Fig. 1). A single suspicious lymph node was also marked with a clip.

After PST completion, the patient underwent re-evaluation with $\mathrm{MRI}$, which indicated complete imaging response to neoadjuvant therapy; the previously described central lesion had been replaced by scarring, and the "non-mass" enhancement had disappeared (Fig. 8).

Approximately one month after PST completion, oncoplastic surgery and SLNB was performed, using the previously placed markers as guidance, and the fhSPECT system to identify the sentinel lymph nodes (SLN). The pathologic report of the specimen confirmed the biopsy results and commented on the multiple foci of the lesion, as well as the presence of several highgrade micropapillary ductal carcinoma in situ (DCIS) sites. The micropapillary DCIS foci surrounding the central lesion were seen to extend up to $0.3 \mathrm{~mm}$ from the excision edge. In total, 5 SLNs were removed, with the pathologist encountering micrometastatic infiltration, and extra-nodal expansion on the previously marked, suspicious node, which was the first SLN, as well. The
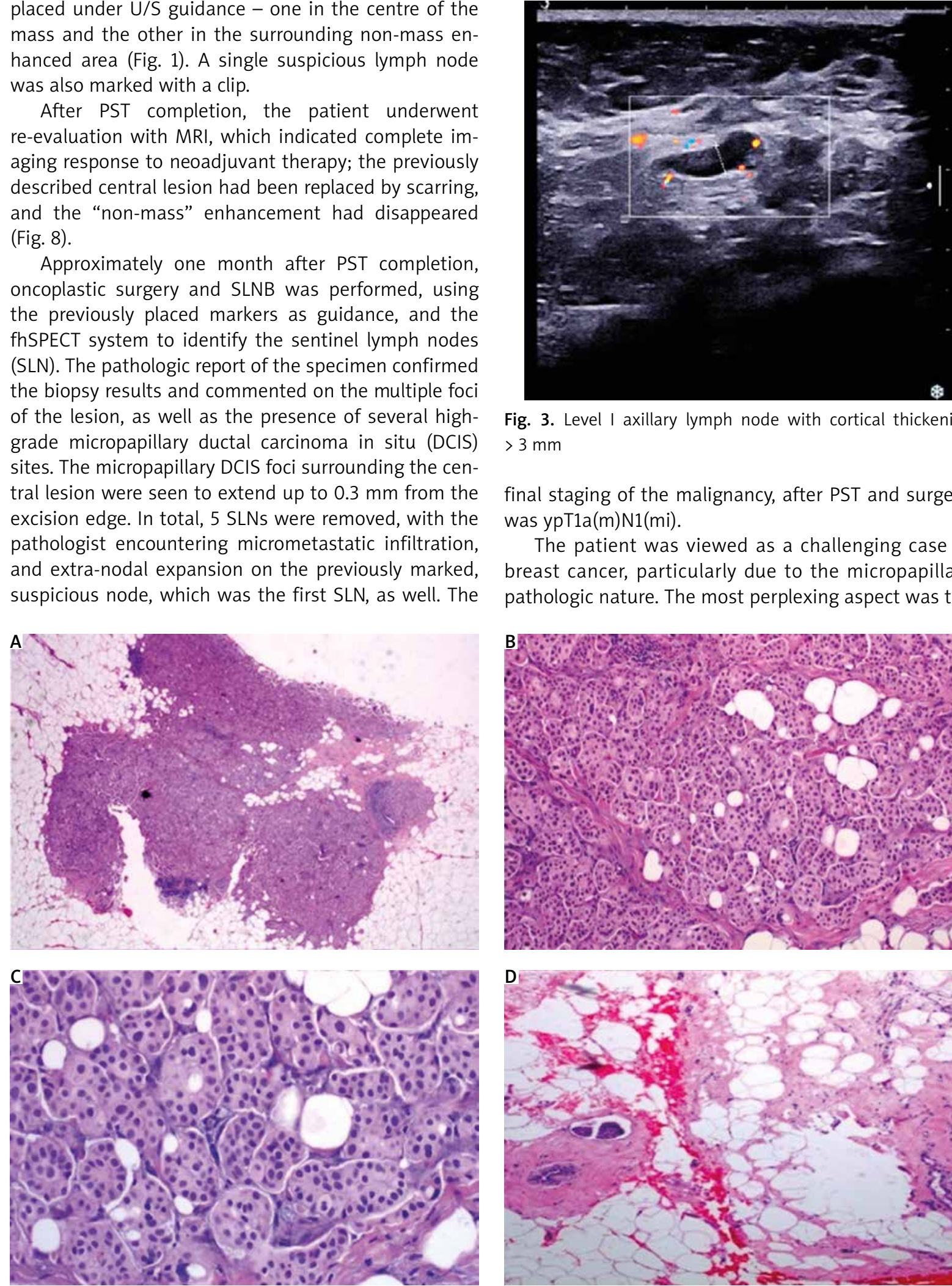

Fig. 3. Level I axillary lymph node with cortical thickening $>3 \mathrm{~mm}$

final staging of the malignancy, after PST and surgery, was ypT1a(m)N1(mi).

The patient was viewed as a challenging case of breast cancer, particularly due to the micropapillary pathologic nature. The most perplexing aspect was the
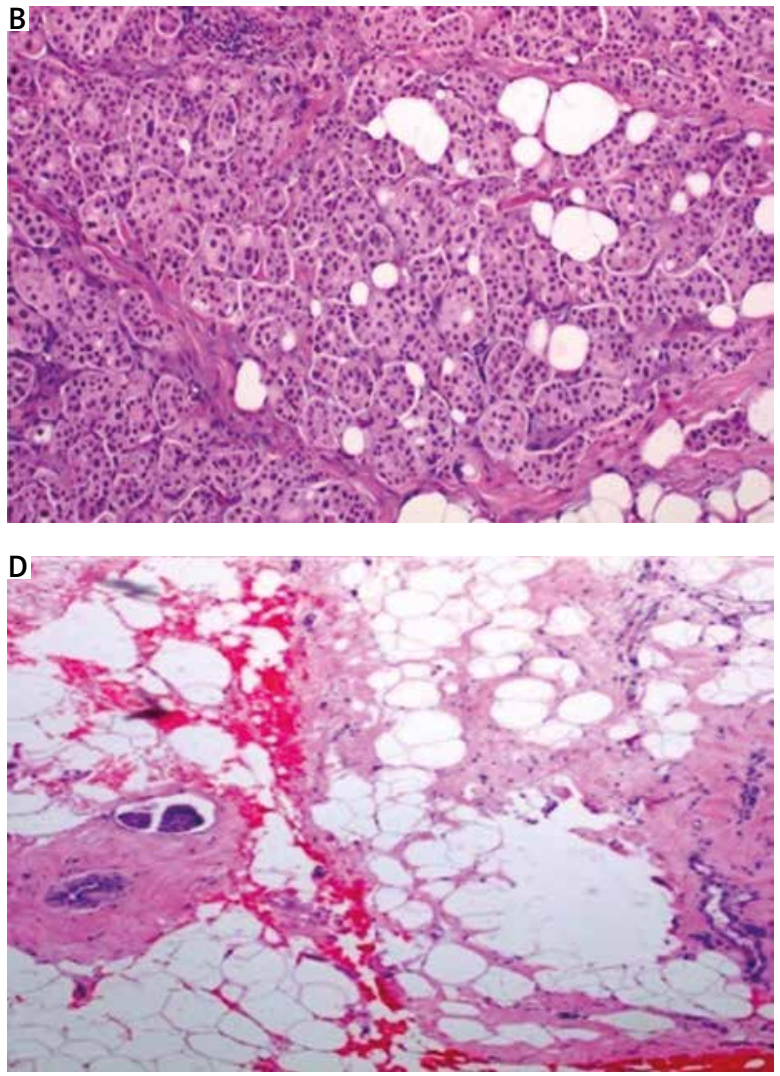

Fig. 4. A) In low magnification, through an atrophic mammary gland a neoplastic population is recognized, infiltrating the remaining ducts; B) the cells are organized in clusters, forming small-sized glandular structures and nests, arranged in a micropapillary pattern, occasionally, a small proportion of them acquires central lumina; C) fibrovascular cores are absent, the neoplastic cells have a moderate amount of eosinophilic cytoplasm and small round nuclei with condensed chromatin and intermediate pleomorphism; D) in another slide of this lesion, lymphovascular emboli are recognized. The morphology is highly suspicious for invasive micropapillary carcinoma, so immunohistochemical markers are performed to establish the diagnosis 

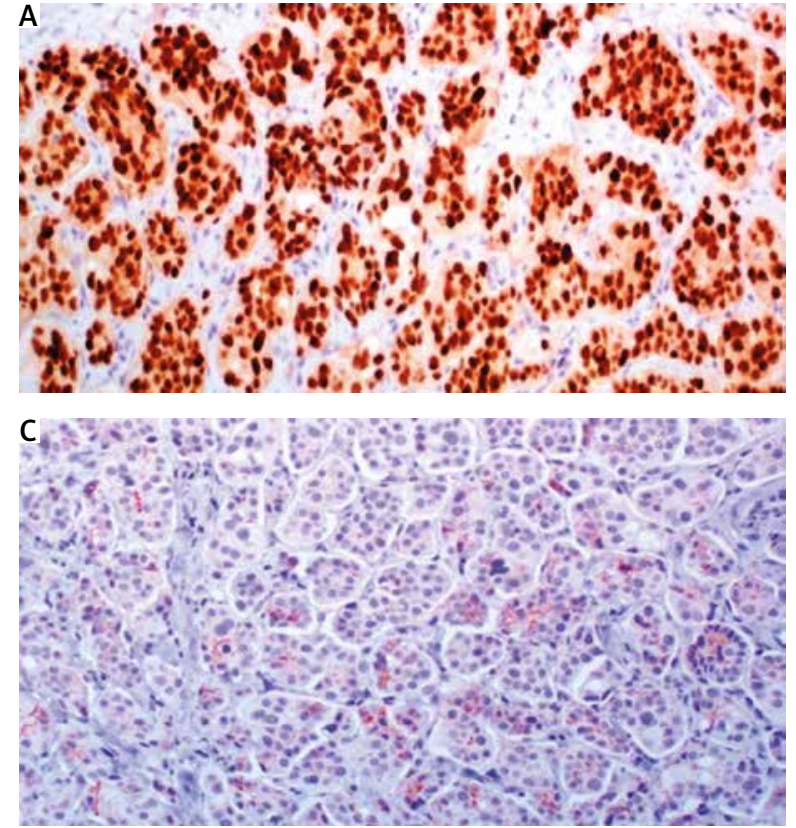

Fig. 5. A) Oestrogen receptor stain is strongly expressed in $95 \%$ of neoplastic cells; B) progesterone receptor is strongly expressed in 60\%; C) cerbB2 score is 1+ (incomplete membranous stain in more than $10 \%$ of neoplastic cells); D) mitotic index Ki67 is $20 \%$

incurrence of micrometastatic disease after PST, a finding that according to most recent guidelines (incorporating the practice-defining findings of the AMAROS trial) [7] is classified as chemoresistant disease. Treatment of ypN1(mi) patients can be either axillary lymph node dissection (ALND) or axillary radiotherapy. However,
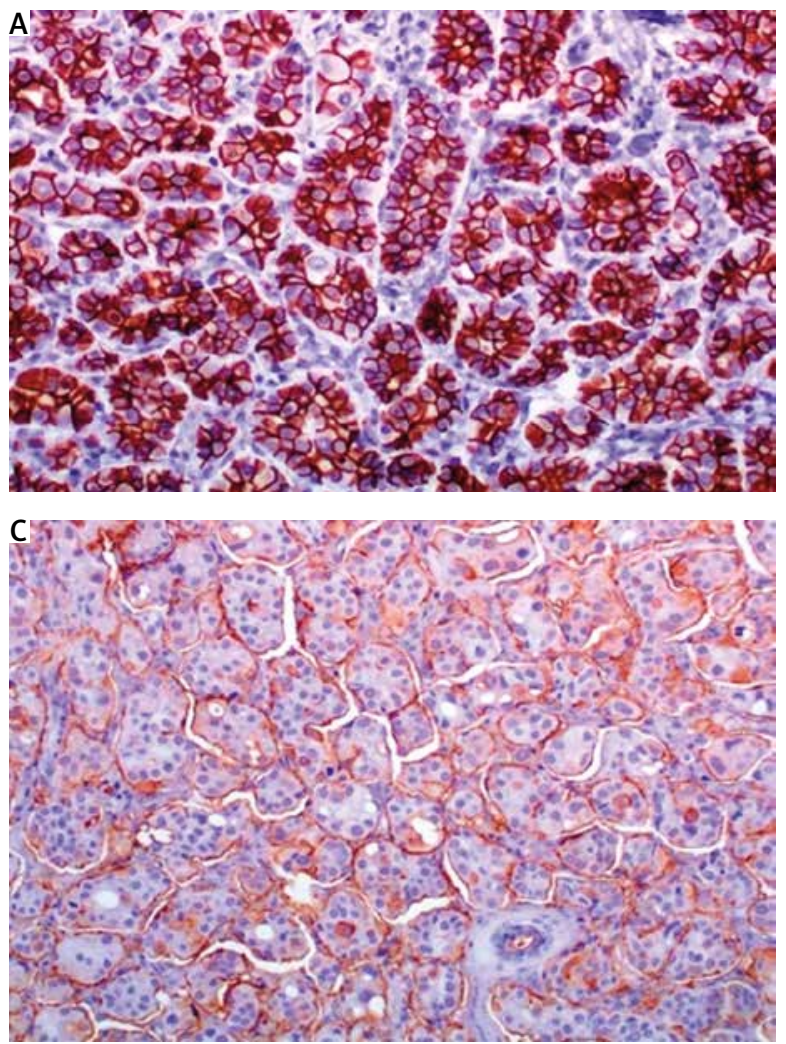
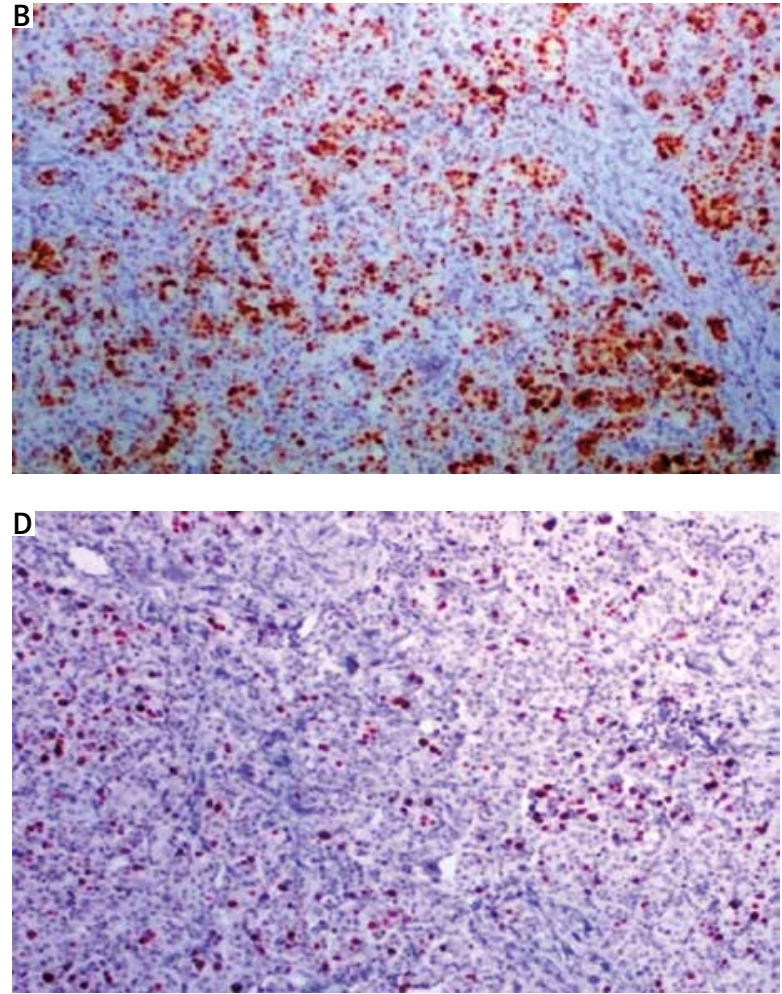

due to the known lymphotropic nature of micropapillary carcinoma, the unsatisfying surgical limits of the oncoplastic surgery, and the presence of micropapillary DCIS, a multidisciplinary team of practitioners decided to perform additionally ALND and modified radical mastectomy.

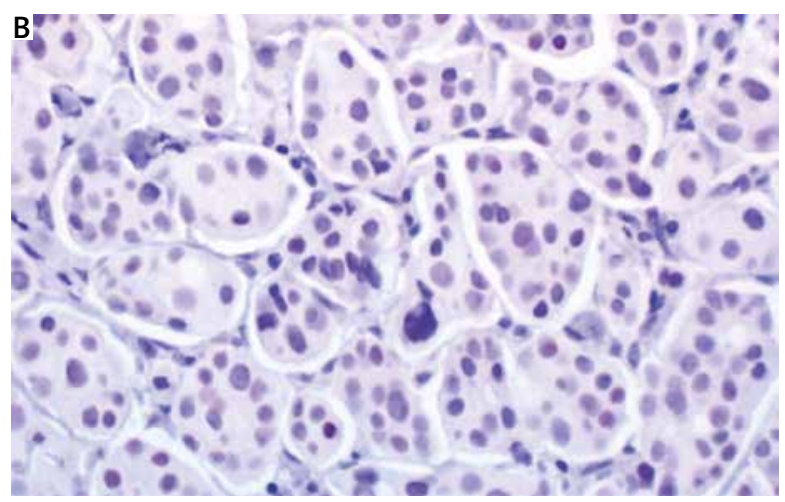

Fig. 6. A) E-cadherin shows an intense, cap-shaped stain; B) due to the eosinophilic appearance, androgen receptor is used in order to exclude an apocrine phenotype. Indeed, androgen receptor stains sporadically; $C$ ) epithelial membrane antigen demonstrates the characteristic pattern of inside out arrangement with the apex of the cells being polarized to the outside surface (reverse polarity) 

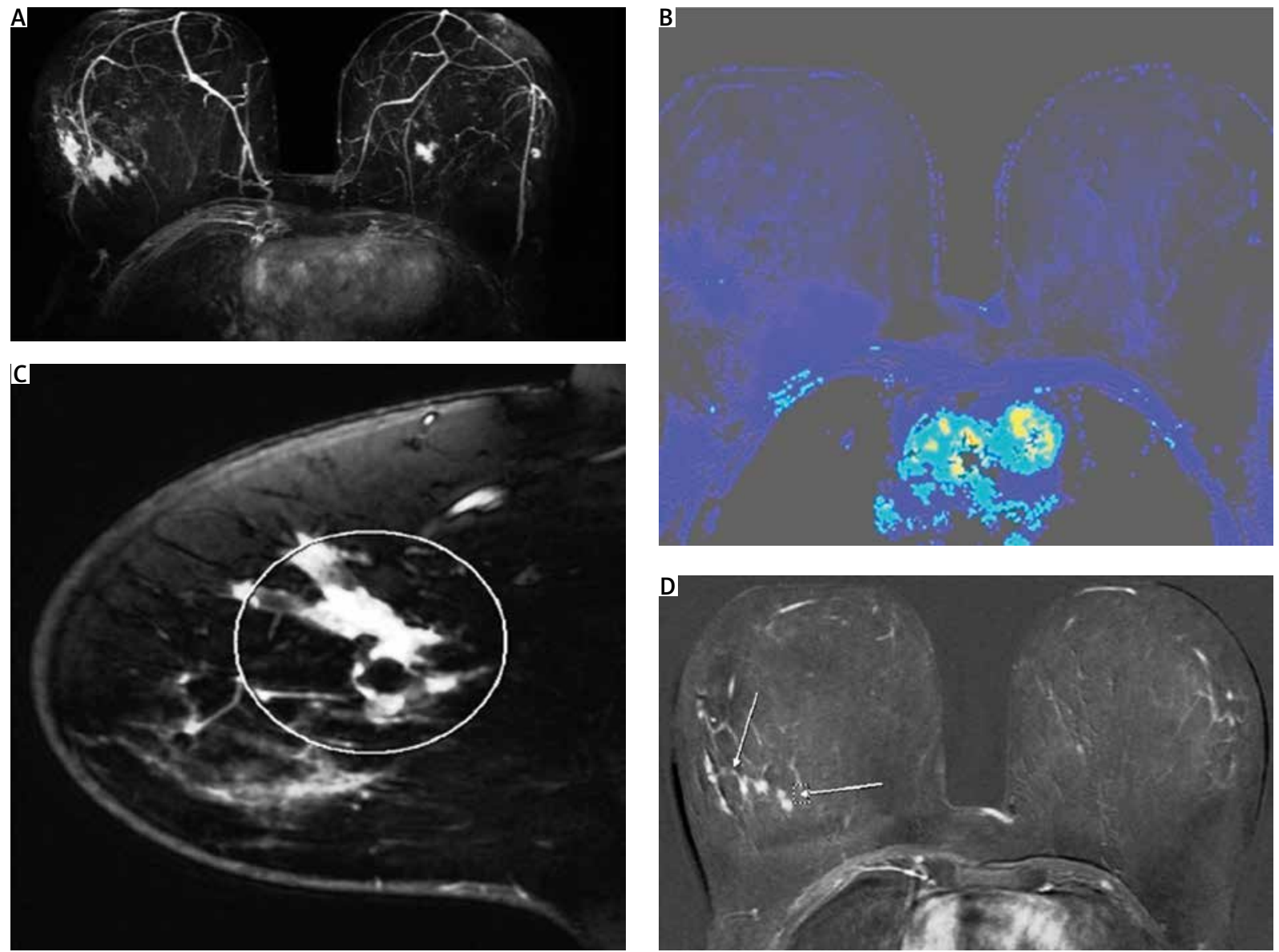

Fig. 7. A-D) Magnetic resonance imaging of the patient. Mass and non-mass enhancement with stellate nodules. The central mass lesion causes pathologic apparent diffusion coefficient values on diffusion-weighted imaging; C-D) the mass is comprised of multiple foci and is of irregular shape

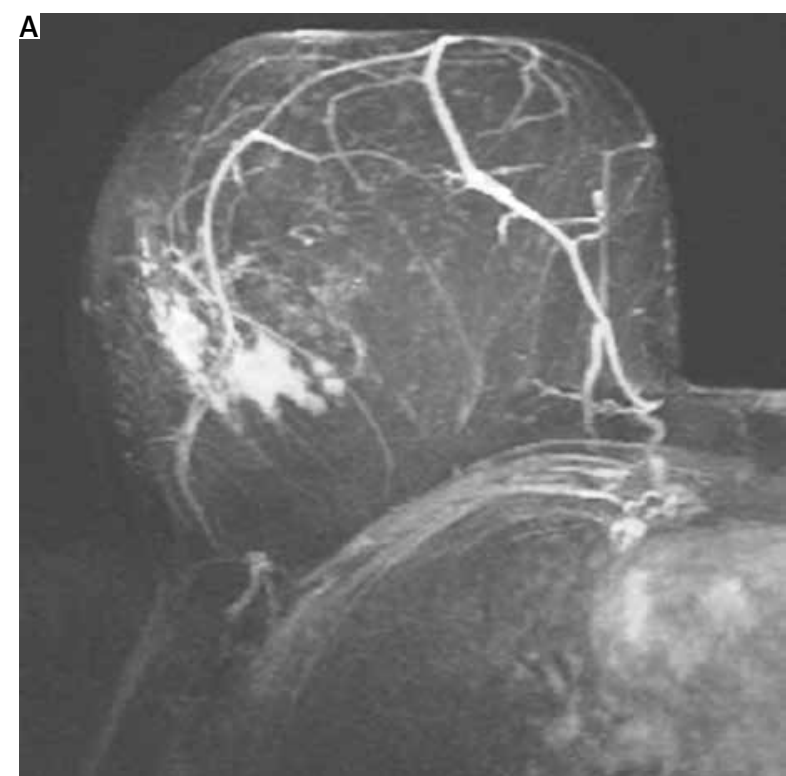

The final pathologic report commented on the presence of multiple (more than 7) scattered foci of infiltrating micropapillary carcinoma, high-grade DCIS lesions of micropapillary histology, and multiple intermedi-

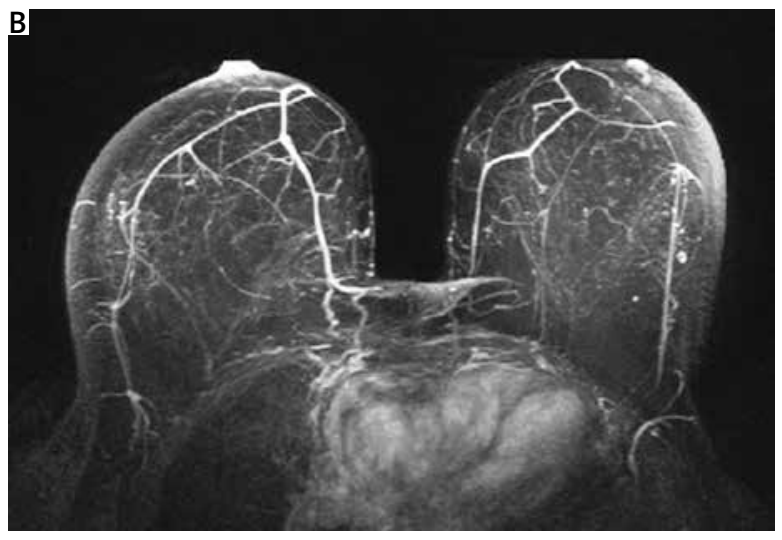

Fig. 8. Magnetic resonance imaging findings consistent with complete radiological response to neoadjuvant chemotherapy

ate-grade LCIS lesions present in the breast tissue. Following mastectomy, adequate surgical margins $(1.5 \mathrm{~cm}$ macroscopically disease free) were ensured, and an additional 10 disease-free lymph nodes were excised on 
ALND, reaching a total of 15 excised lymph nodes when added to those of SLNB. The postoperative course was uncomplicated, and the patient was discharged under standard postoperative follow-up observation.

\section{Discussion}

In recent years, breast carcinoma has been rising in prevalence, reaching the status of the most common malignancy worldwide. Heterogenicity is a well-recognized aspect of breast carcinoma, with more than 28 distinct histological subtypes being described in the literature. Invasive micropapillary carcinoma is a well-defined histopathological subtype, rarely encountered, which is found in approximately $3-6 \%$ of all invasive carcinomas of the breast [8]. Diagnosis of IMPC is done by histological study and is based on some characteristic structural findings in the breast tissue. Clusters of atypical cells are presented in a papillary or pseudopapillary arrangement, lacking a fibrovascular core, and arranged in a peculiar manner, with evidence of reversed cellular polarity [9-12]. This change in polarity is thought to be a result of complex molecular changes during the process of carcinogenesis, mainly regarding anchoring proteins and proteinases, much of which can also account for the trend in metastasizing [13-18].

Micropapillary breast carcinoma is best known for its tendency for lymphovascular invasion and lymph node metastases. The prevalence of nodal invasion at presentation is remarkably elevated for IMPC, reaching rates of $75 \%$, and even $95 \%$ in some case series [19-21]. Several studies have determined that the frequency of nodal involvement in IMPC, is significantly higher compared to non-specific type (NST) carcinoma, as well as the mean number of disease-positive lymph nodes excised in patients. Research also suggests that IMPC tumours are larger on average and can be associated with higher frequency of local recurrence than other histological types [22-25]. While it was once also believed that the micropapillary pattern was associated with worse outcomes and prognosis, recent meta-analyses on the subject suggest that when comparing IMPC breast carcinoma patients with NST ones in a propensity-matched manner, overall survival rates and disease-free survival did not differ significantly [25-29]. The previously observed inferior outcomes are now thought to be a result of the more extensive nodal involvement at presentation when it comes to IMPC patients, instead of the histology itself, because nodal status and lymphovascular infiltration are both independent prognostic factors for the survival of breast cancer patients.

Reports on IMPC also suggest that on average, patients diagnosed with IMPC, present with tumours of increased size and higher stage at diagnosis, when compared with other types of breast carcinoma, most nota- bly NST [28-30]. This observed difference is thought to be caused by a more rapid growth profile, evident by certain variations regarding growth factors as well as the histology's metastasizing and lymphotropic tendency. Studies on the immunochemistry profile of IMPC do not point to any significant alterations when compared to other histological subtypes of breast cancer [31].

The occurrence of pure IMPC is a rare phenomenon, found in approximately 1-2\% of breast carcinomas [32]. Authors suggest a cut-off percentile value of $75 \%$ IMPC component, in order to histologically identifying a carcinoma as pure IMPC [33]. On the other hand, the presence of micropapillary component within NST tumours is a more frequent occurrence, and much deliberation has been made on its clinical significance. Multiple researchers have reported an association between the micropapillary element being present within a tumour and worse prognosis, as well as once again a lymphotropic pattern [22, 34-38]. The presence of micropapillary DCIS foci, as found in our case, has also been frequently studied in the literature. Authors have found that the presence of micropapillary DCIS is associated with an increase in recurrence rates, higher-grade tumours, and increased tumour size at diagnosis. The presence of micropapillary DCIS foci, compared to the presence of simple, non-characterized DCIS, was also found to be associated with worse prognosis, higher recurrence rates, and increased incidence of lymphovascular invasion $[39,40]$. As mentioned earlier, it should be noted that worse prognosis seems to be attributed to the increased frequency of lymph node invasion rather than the histology itself, and therefore most comparative studies that are done in a non-adjusted manner should not be interpreted as evidence of a unique histopathologic aggressiveness other than that of increased lymphovascular tropism.

Imaging studies of IMPC harbour certain difficulties in their interpretation. The mammographic appearance is usually nonspecific for the lesion type, and lesions are described as irregular, spiculated masses frequently combined with microcalcification. Notably, researchers comment that standard mammography is susceptible to under-evaluation of the true extent of the disease when it comes to IMPC and can even lead to false negative results in $12 \%$ of cases [41-43]. On U/S, IMPC is revealed as a hypoechoic mass with irregular margins; however, reported false negative rates reaching $47 \%$ as well as comparison with other imaging modalities seem to suggest that U/S is ineffective in estimating the breast lesion [41]. On the contrary, axillary U/S evaluation in IMPC patients offers valuable insights in the nodal status and frequently reveals signs of nodal infiltration, such as cortical thickening. This was also the case in our patient, in whom a single suspicious node was detected and preoperatively marked, which was ultimately reported as infiltrated in the final pathologic 
report. Evaluation by MRI seems to be the gold standard regarding accurate depiction of the true extent of IMPC [39, 41, 44-46]. Lesions are seen as irregular, spiculated masses with rapid enhancement and delayed washout. A solitary enhanced mass is the usual form of presentation; however, non-mass enhancement similar to what was seen in our case can be found in up to $38.9 \%$ of cases [46]. This area of non-mass enhancement can be indicative of local lymphovascular invasion and the presence of DCIS loci. Micropapillary carcinoma presenting as a diffuse lesion rather than a solitary tumour is best depicted via MRI, although there are reports of missed disease.

To date, there are no surgical guidelines specifically for the treatment of IMPC. In the past, surgeons thought the diagnosis of IMPC or the presence of micropapillary component within a tumour constituted sufficient reason for radical axillary excision. Despite a lack of randomized trials regarding IMPC treatment, data on survival, recurrence, and prognosis no longer support the use of any approach different than that outlined in current guidelines on breast surgery [28, 29, 32, 47]. Studies also suggest that most cases of IMPC are treated with mastectomy techniques rather than breast conserving techniques; however, this is also done in accordance with the tumour stage at diagnosis rather than the histological typing. Nonetheless, it remains a point of importance for breast surgeons to be more vigilant and thorough in axillary evaluation preoperatively, as well as post-PST treatment. Our case is a prime example of the gravity of proper evaluation of the lymphatic aspect of IMPC. Utilization of axillary U/S and biopsy markers was crucial in locating and excising a suspicious lymph node, which ultimately led to escalation of the surgical treatment, in accordance with guidelines on management of the axilla in post-PST patients.

\section{Conclusions}

Our case report outlines the importance of awareness of histological subtypes in breast cancer by focusing on a case report of IMPC. The breast surgeon must be aware of the lymphotropic behaviour of this subtype and the high prevalence of lymph node involvement in such patients, and therefore focus on rigorous axillary assessment. One must not forget that, despite having a more aggressive biological profile, IMPC has demonstrated no difference in survival when compared to other histological subtypes, and treatment should conform to international guidelines with an emphasis on nodal staging.

\section{Disclosure}

The authors report no conflict of interest.

\section{References}

1. Fisher ER, Gregorio R, Redmond C, Dekker A, Fisher B. Pathologic findings from the national surgical adjuvant breast project (protocol no. 4). II. The significance of regional node histology other than sinus histiocytosis in invasive mammary cancer. Am J Clin Pathol 1976; 65: 21-30.

2. Siriaunkgul S, Tavassoli FA. Invasive micropapillary carcinoma of the breast. Mod Pathol 1993; 6: 660-662.

3. Yang YLYL, Liu BBBB, Zhang X, Fu L. Invasive micropapillary carcinoma of the breast: an update. Arch Pathol Lab Med 2016; 140: 799-805.

4. Stranix JTT, Kwa MJJ, Shapiro RLL, Speyer JLL. Invasive micropapillary carcinoma of the male breast: Case report and review of the literature. Cancer Treat Commun 2015; 3: 44-49.

5. Vingiani A, Maisonneuve P, Dell'Orto P, et al. The clinical relevance of micropapillary carcinoma of the breast: A case-control study. Histopathology 2013; 63: 217-224.

6. Coyle EA, Taj H, Comba I, Vasquez J, Zayat V. Invasive micropapillary carcinoma: a rare case of male breast cancer. Cureus 2020; 12: e10571.

7. Gandhi A, Coles C, Makris A, et al. Axillary surgery following neoadjuvant chemotherapy - multidisciplinary guidance from the Association of Breast Surgery, Faculty of Clinical Oncology of the Royal College of Radiologists, UK Breast Cancer Group, National Coordinating Committee for Breast. Clin Oncol 2019; 31: 664-668.

8. Hao S, Zhao YY, Peng JJ, et al. Invasive micropapillary carcinoma of the breast had no difference in prognosis compared with invasive ductal carcinoma: a propensity-matched analysis. Sci Reports 2019; 9: 286.

9. Kim MJ, Gong G, Joo HJ, Ahn SH, Ro JY. Immunohistochemical and clinicopathologic characteristics of invasive ductal carcinoma of breast with micropapillary carcinoma component. Arch Pathol Lab Med 2005; 129: 1277-1282.

10. Wong SI, Cheung H, Tse GMK. Fine needle aspiration cytology of invasive micropapillary carcinoma of the breast: a case report. Acta Cytol 2000; 44: 1085-1089.

11. Ongürü O, Deveci S, Günhan O, Öngürü Ö, Deveci S, Günhan Ö. Cytological findings of invasive micropapillary carcinoma of the breast: a report of two cases. Cytopathology 2002; 13: 160-163.

12. Cui ZQ, Feng JH, Zhao YJ. Clinicopathological features of invasive micropapillary carcinoma of the breast. Oncol Lett 2015; 9: 1163-1166.

13. Onder S, Fayda M, Karanlık H, et al. Loss of ARID1A expression is associated with poor prognosis in invasive micropapillary carcinomas of the breast: a clinicopathologic and immunohistochemical study with longterm survival analysis. Breast J 2017; 23: 638-646.

14. Doublier S, Belisario DC, Polimeni M, et al. HIF-1 activation induces doxorubicin resistance in MCF7 3-D spheroids via P-glycoprotein expression: a potential model of the chemo-resistance of invasive micropapillary carcinoma of the breast. BMC Cancer 2012; 12.

15. Meng F, Liu B, Xie G, et al. Amplification and overexpression of PSCA at 8 q24 in invasive micropapillary carcinoma of breast. Breast Cancer Res Treat 2017; 166: 383-392.

16. Lewis GD, Xing Y, Haque W, et al. The impact of molecular status on survival outcomes for invasive micropapillary carcinoma of the breast. Breast J 2019; 25: 1171-1176.

17. Ren M, Liu F, Zhu Y, et al. Absence of caveolin-1 expression in carcinomaassociated fibroblasts of invasive micropapillary carcinoma of the breast predicts poor patient outcome. Virchows Arch 2014; 465: 291-298.

18. Liu F, Lang R, Wei J, et al. Increased expression of SDF-1/CXCR4 is associated with lymph node metastasis of invasive micropapillary carcinoma of the breast. Histopathology 2009; 54: 741-750.

19. Vingiani A, Maisonneuve P, Dell'Orto $P$, et al. The clinical relevance of micropapillary carcinoma of the breast: A case-control study. Histopathology 2013; 63: 217-224.

20. Meng $\mathrm{X}, \mathrm{Ma} \mathrm{H}$, Yin $\mathrm{HH}$, et al. Nomogram predicting the risk of locoregional recurrence after mastectomy for invasive micropapillary carcinoma of the breast. Clin Breast Cancer 2021; 21: e368-e376.

21. Wang R, Li N, Wang XJ, et al. Differences in the clinicopathological characteristics of pure and mixed invasive micropapillary breast carcinomas from eastern China. Ann Transl Med 2021; 9: 412.

22. Bal A, Joshi K, Sharma SC, Das A, Verma A, Wig JD. Prognostic significance of micropapillary pattern in pure mucinous carcinoma of the breast. Int I Surg Pathol 2008; 16: 251-256. 
23. Zhang LT, Gao DC, Lu XM. Pathologic and clinical features of invasive micropapillary carcinoma of breast. Zhonghua Bing Li Xue Za Zhi 2007; 36: 838-839.

24. Tang SL, Yang JQ, Du ZG, et al. Clinicopathologic study of invasive micropapillary carcinoma of the breast. Oncotarget 2017; 8: 42455-42465.

25. Wu Y, Zhang N, Yang Q. The prognosis of invasive micropapillary carcinoma compared with invasive ductal carcinoma in the breast: a metaanalysis. BMC Cancer 2017; 17: 839.

26. Hao S, Zhao YY, Peng JJ, et al. Invasive micropapillary carcinoma of the breast had no difference in prognosis compared with invasive ductal carcinoma: a propensity-matched analysis. Sci Rep 2019; 9: 286.

27. Shi WB, Yang LJ, Hu X, Zhou J, Zhang Q, Shao ZM. Clinico-pathological features and prognosis of invasive micropapillary carcinoma compared to invasive ductal carcinoma: a population-based study from China. PLoS One 2014; 9.

28. Li G, Yang S, Yao J, et al. Invasive micropapillary carcinoma of the breast had poor clinical characteristics but showed no difference in prognosis compared with invasive ductal carcinoma. World J Surg Oncol 2016; 14: 207.

29. Chen H, Wu K, Wang M, Wang F, Zhang M, Zhang P. Invasive micropapillary carcinoma of the breast has a better long-term survival than invasive ductal carcinoma of the breast in spite of its aggressive clinical presentations: a comparison based on large population database and case - control analysis. Cancer Med 2017; 6: 2775-2786.

30. Liu F, Yang M, Li Z, et al. Invasive micropapillary mucinous carcinoma of the breast is associated with poor prognosis. Breast Cancer Res Treat 2015; 151: 443-451.

31. Luna-Moré S, Casquero S, Pérez-Mellado A, Rius F, Weil B, Gornemann I. Importance of estrogen receptors for the behavior of invasive micropapillary carcinoma of the breast. Review of 68 cases with follow-up of 45 . Pathol Res Pract 2000; 196: 35-39.

32. Chen AC, Paulino AC, Schwartz MR, et al. Population-based comparison of prognostic factors in invasive micropapillary and invasive ductal carcinoma of the breast. Br J Cancer 2014; 111: 619-622.

33. Kaya C, Uçak R, Bozkurt E, et al. The impact of micropapillary component ratio on the prognosis of patients with invasive micropapillary breast carcinoma. J Investig Surg 2020; 33: 31-39.

34. Ranade AC, Batra R, Sandhu G, Chitale RA, Balderacchi J. Clinicopathological evaluation of 100 cases of mucinous carcinoma of breast with emphasis on axillary staging and special reference to a micropapillary pattern. J Clin Pathol 2010; 63: 1043-1047.

35. Wu Y, Zhang N, Yang Q. The prognosis of invasive micropapillary carcinoma compared with invasive ductal carcinoma in the breast: a metaanalysis. BMC Cancer 2017; 17: 1-9.

36. Cima L, Mirabassi N, Sartori C, et al. Mixed invasive apocrine papillary/ micropapillary carcinoma of the breast: another brick in the triple-negative wall. Int J Surg Pathol 2020; 29: 420-426.

37. Sun P, Zhong Z, Lu Q, et al. Mucinous carcinoma with micropapillary features is morphologically, clinically and genetically distinct from pure mucinous carcinoma of breast. Mod Pathol 2020; 33: 1945-1960.

38. Xu X, Bi R, Shui R, et al. Micropapillary pattern in pure mucinous carcinoma of the breast - does it matter or not? Histopathology 2019; 74: 248-255.

39. Lee YS, Mathew J, Dogan BE, Resetkova E, Huo L, Yang WT. Imaging features of micropapillary DCIS: correlation with clinical and histopathological findings. Acad Radiol 2011; 18: 797-803.

40. Evers K. Significance of finding micropapillary DCIS on core needle biopsy 2011. Acad Radiol 2011; 18: 795-796.

41. Alsharif S, Daghistani R, Kambero lu EA, Omeroglu A, Meterissian S, Mesurolle B. Mammographic, sonographic and MR imaging features of invasive micropapillary breast cancer. Eur J Radiol 2014; 83: 1375-1380.

42. Lee YS, Mathew J, Dogan BE, Resetkova E, Huo L, Yang WT. Imaging features of micropapillary DCIS: correlation with clinical and histopathological findings. Acad Radiol 2011; 18: 797-803.

43. Jones KN, Guimaraes LS, Reynolds CA, Ghosh K, Degnim AC, Glazebrook $\mathrm{KN}$. Invasive micropapillary carcinoma of the breast: Imaging features with clinical and pathologic correlation. Am J Roentgenol 2013; 200: 689-695.

44. Lim HS, Kuzmiak CM, Jeong SI, et al. Invasive micropapillary carcinoma of the breast: MR imaging findings. Korean J Radiol 2013; 14: 551-558.
45. Adrada B, Arribas E, Gilcrease M, Yang WT. Invasive micropapillary carcinoma of the breast: Mammographic, sonographic, and MRI features. Am J Roentgenol 2009; 193: 58-63.

46. Han CH, Yao WG, He J, Gao ZB, Hu HJ. MRI and the pathology of breast invasive micropapillary carcinoma. Oncol Lett 2020; 20: 2811-2819.

47. Terando AM, Agnese DM, Holmes DR. Treatment and prognosis of rare breast cancers. Ann Surg Oncol 2015; 22: 3225-3229. 